\title{
MODELAGEM PARA A ESTIMATIVA DA ORIENTAÇÃO DE LINHAS DE PLANTIO DE CAFEEIROS ${ }^{1}$
}

\author{
KLERISSON M. G. OLIVEIRA ${ }^{2}$, LUIZ G. DE CARVALHO ${ }^{3}$, LUIZ A. LIMA ${ }^{4}$, \\ RAPHAELA C. C. GOMES ${ }^{5}$
}

\begin{abstract}
RESUMO: A quantidade de radiação solar interceptada por uma cultura é uma importante variável meteorológica que determina o crescimento e o desenvolvimento de uma cultura agrícola e, dentre os sistemas de produção, a orientação das linhas de plantio poderá ser um componente bastante relevante quanto à interceptação da radiação solar direta $(\mathrm{Rd}) \mathrm{em}$ ambas as faces. Portanto, este trabalho propõe como objetivo um modelo para recomendar a orientação adequada das linhas de cafeeiro na implantação, baseando-se naquela a proporcionar uma uniformidade na quantidade de Rd interceptada e acumulada durante o ciclo agrícola da cultura, em ambas as faces da linha de plantio. Para o teste do modelo, foram coletados dados de produção em uma área experimental de café irrigado por pivô central, na Fazenda São Thomé, em Pirapora-MG, em plantas alinhadas nas seguintes orientações, em relação ao norte geográfico, positivo no sentido horário e negativo ao contrário: $-90^{\circ},-45^{\circ}, 0^{\circ}$ e $45^{\circ}$. Os resultados indicaram que as melhores orientações para a produtividade foram, sucessivamente, as de $-45^{\circ}$ e $0^{\circ}$, enquanto o modelo proposto estimou a orientação de $-24^{\circ} 16^{\prime}$ como sendo a melhor para o plantio das linhas de cafeeiro.
\end{abstract}

PALAVRAS-CHAVE: café, sistema de cultivo, radiação solar.

\section{MODELLING FOR ESTIMATING THE ORIENTATION OF COFFEE PLANTING ROWS}

ABSTRACT: The quantity of solar radiation intercepted by a crop is an important meteorological variable that determines the growth and development of a crop and among the production systems, the orientation of planting rows could be a very important component regarding the interception of direct solar radiation ( $\mathrm{Rd})$ on both faces. Thus the objective of this study proposes a model to recommend the adequate orientation of the planting rows of coffee in the deployment, based on that providing uniformity in quantity of intercepted and accumulated $\mathrm{Rd}$ during the crop cycle on both faces of the planting row. In order to test the model, production data were collected in an experimental area of the coffee crop irrigated by a center pivot in San Thomé Farm on Pirapora, state of Minas Gerais, Brazil, being this planting rows oriented in relation to geographic north, clockwise positive and negative rather, by $45^{\circ},-90^{\circ},-45^{\circ}$, and $0^{\circ}$. The results indicated that the best orientations for productivity were, successively, the $-45^{\circ}$ and $0^{\circ}$, while the proposed model estimates the orientation of $-24^{\circ} 16^{\prime}$ as the best for planting rows of coffee crop.

KEYWORDS: coffee, cultive system, solar radiation.

\footnotetext{
${ }^{1}$ Extraído da dissertação do primeiro autor.

${ }^{2}$ Graduado e Mestre em Engenharia Agrícola, área de concentração Engenharia de Água e Solo, Universidade Federal de Lavras UFLA, Departamento de Engenharia, Lavras - MG, klerissonmilton@gmail.com.

${ }^{3}$ Prof. Doutor, Universidade Federal de Lavras - UFLA, Departamento de Engenharia, Núcleo de Agrometeorologia e Climatologia, Lavras - MG, lgonsaga@deg.ufla.br.

${ }^{4}$ Prof. Phd, Universidade Federal de Lavras - UFLA, Departamento de Engenharia, Núcleo Engenharia de Água e Solo, Lavras MG, lalima@deg.ufla.br.

${ }^{5}$ Engenheira e Mestre em Engenharia Agrícola, área de concentração em Ambiência Animal, Universidade Federal de Lavras UFLA, Departamento de Engenharia, Doutoranda pela Universidade Federal de Goiás, Goiânia - GO, raphachris@gmail.com.

Recebido pelo Conselho Editorial em: 10-6-2010

Aprovado pelo Conselho Editorial em: 22-11-2011
} 


\section{INTRODUÇÃO}

Historicamente, a cafeicultura brasileira tem relevante importância econômica e social. Segundo dados da CONAB (2010) a produção nacional de café atingiu 39,5 milhões de sacas de café beneficiado em 2009, sendo Minas Gerais responsável por 50,4\% dessa produção. Numerosas são as propostas de sistemas de produção inovadores que buscam aumento da competitividade; contudo, como envolvem investimentos e alterações nas práticas tradicionalmente utilizadas, é natural que existam dúvidas quanto ao seu mérito econômico, quando consideradas as características peculiares de uma dada região produtora (OLIVEIRA et al., 2010). Dentre esses sistemas de produção, a orientação das linhas de plantio poderá ser um componente bastante relevante quanto à interceptação da radiação solar direta em ambas as faces. A radiação solar interceptada por uma cultura é uma importante variável meteorológica que determina sua transpiração e fotossíntese, necessitando ser avaliada e mais bem estudada (ANGELOCCI et al., 2008). De acordo com as citações de BRAUN et al. (2007) e TATAGIBA et al. (2010), sabe-se que o cafeeiro é uma planta $\mathrm{C} 3$, ou seja, é uma planta de ambiente sombreado, que possui adaptações fisiológicas e morfológicas para esse tipo de ambiente. PEZZOPANE et al., (2005) e PEZZOPANE et al. (2007) realizaram um estudo efetuando medições da radiação solar e do saldo de radiação em cafeeiros cultivados a pleno sol e consorciados com bananeira e observaram atenuação média dos valores de radiação solar no sistema consorciado da ordem de $21 \%$, com variação mensal de $16 \%$ a $27 \%$. Semelhantemente, RIGHI et al. (2007) estudaram a influência da radiação solar sobre cafeeiro cultivado dentro e ao lado de uma plantação de seringueira; as plantas toleraram diminuição de 50\% na disponibilidade de radiação solar sem sofrer reduções no crescimento e IAF.

Uma questão agravante no que se refere à intensidade de radiação solar interceptada é o efeito da escaldadura em cafeeiro, pois, segundo SANTINATO et al. (1996), a escaldadura, que é a descoloração foliar, ocorre notadamente na face da linha de plantio voltada para o "sol da tarde" em regiões de cerrado. No cultivo do cafeeiro, no qual tradicionalmente se obedece ao plantio em nível, ou mesmo em plantios circulares irrigados sob pivô central, tem-se observado, mesmo que empiricamente, sem comprovações científicas, considerável variação da produtividade nas diferentes faces da linha de plantio. Essa heterogeneidade pode ser atribuída aos diferentes níveis de energia radiante interceptada por faces opostas numa linha de plantio.

A topografia é outro fator determinante na intensidade e na qualidade da radiação interceptada. Nesta linha, pode ser citado o trabalho desenvolvido por TURCO \& RIZZATTI (2006) em que estudaram um modelo matemático para estimar a radiação solar diária sobre superfícies com diferentes exposições e declividades. A variabilidade espacial da radiação solar em áreas de diferentes inclinações e exposições foi estudada por TOVAR-PESCADOR et al. (2006), e eles verificaram que as variações do relevo são altamente significativas na distribuição da radiação solar junto à superfície terrestre. Outro estudo envolvendo a influência da topografia, presença de nuvens e heterogeneidade de florestas foi apresentado por OLIPHANT et al. (2006) em Indiana-USA, os quais notaram a alta sensibilidade da radiação à topografia e decréscimo na transmissividade atmosférica quando na ausência de nuvens. DONATELLI et al. (2006) desenvolveram um modelo que permite calcular a radiação solar que atinge a superfície terrestre sob diversas condições meteorológicas e superfícies inclinadas, tornando o processo ágil e eficaz.

GANIS (1997) apresentou um modelo simples para a estimativa da penetração da radiação fotossinteticamente ativa dentro do dossel vegetativo da cultura do milho, sendo a modelagem uma boa alternativa para a orientação da linha de plantio, visando à melhor uniformidade da penetração da radiação fotossintética em todo o dossel da cultura. Por sua vez, CUNHA \& VOLPE (2010), especificamente para o cafeeiro variedade Obatã IAC 1669-20, estudaram a influência de três diferentes alinhamentos de plantio no terço superior da copa de cafeeiro e o alinhamento próximo ao movimento aparente diário do Sol $\left(81^{\circ}-261^{\circ}\right)$ revelou maior eficiência na interceptação da radiação fotossinteticamente ativa. 
BOSCH et al. (2009) evidenciaram a importância de se estudar a influência da turbidez atmosférica na incidência da radiação solar junto à superfície terrestre, realizando um estudo sobre a qualidade da radiação fotossinteticamente ativa. Semelhantemente, LIMA et al. (2007) realizaram estudo avaliando a influência de nuvens, água precipitável e precipitação pluvial na radiação solar que atinge a superfície terrestre, verificando que estes são elementos primordiais, tanto na transmitância atmosférica, afetando a radiação direta $(\mathrm{Rd})$, como a radiação difusa, sendo ambas componentes da radiação solar incidente à superfície, por isso sendo muito comum expressá-la como radiação global.

Diante do exposto, estabeleceu-se a hipótese para o presente trabalho de que, quantificando a interceptação da Rd em ambas as faces de linhas de plantio do cafeeiro sob diferentes orientações, identifica-se a orientação que promove distribuição uniforme de energia na cultura a fim de minimizar a escaldadura das folhas. Portanto, sob esta hipótese é que se desenvolveu o presente modelo, de forma a subsidiar o cafeicultor na implantação da lavoura de café, com relação à orientação das linhas de plantio.

\section{MATERIAL E MÉTODOS}

\section{Desenvolvimento do modelo}

O desenvolvimento do modelo proposto foi com base na metodologia de PALTRIDGE \& PLATT (1976), a qual também é apresentada por ALVES (1981), seguindo a sequência de equações descritas a seguir, com implementações necessárias para o presente trabalho.

Sabendo-se que a radiação difusa é uma componente da radiação solar e por esta incidir à superfície, independentemente de sombreamento, com mudança apenas na intensidade, o presente estudo foi realizado adotando-se apenas a influência da radiação solar direta ( $R d)$. Pela hipótese levantada, a preocupação somente com Rd, visa a uniformizar a interceptação desta componente nas duas faces de exposição da linha de plantio, justamente por ser o cafeeiro uma cultura de ambiente sombreado, como citam BRAUN et al. (2007) e TATAGIBA et al. (2010), pois, no campo, não sendo possível cultivar em ambiente totalmente sombreado, ao menos que haja uniformidade da Rd incidente sobre a cultura.

Assim, o modelo de PALTRIDGE \& PLATT (1976) foi adotado considerando apenas a estimativa da interceptação da $\mathrm{Rd}$, a qual é possível de ser estimada para diferentes localidades e ainda sob diferentes exposições e inclinações da superfície (eq.(1)).

$$
\mathrm{I}_{0 \mathrm{i}}=\mathrm{I}_{0} \cos (\theta)+\alpha\left(\mathrm{I}_{0} \cos (\mathrm{Z})\right)\left[1-\cos ^{2}\left(\frac{\mathrm{i}}{2}\right)\right]
$$

em que, $\mathrm{I}_{0 \mathrm{i}}$ - Rd sobre a superfície inclinada, $\mathrm{W} \mathrm{m} \mathrm{m}^{-2} ; \mathrm{I}_{0}-\mathrm{Rd}$ sobre a superfície plana, $\mathrm{W} \mathrm{m}^{-2} ; \theta$ ângulo de incidência da $\mathrm{Rd}$ com a normal à superfície, rad; $\mathrm{i}$ - ângulo de inclinação da superfície, rad; $\alpha$ - índice de refletividade da superfície, adimensional, e Z - ângulo zenital, rad.

Para a estimativa de $\mathrm{I}_{0}$, utilizou-se a expressão de BROOKS (1959), eq.(2), na qual é necessária a determinação da transmitância atmosférica, sendo esta interferente à chegada da radiação solar à superfície terrestre, como descrevem LIMA et al. (2007) e BOSCH et al. (2009).

$$
\mathrm{I}_{0}=\mathrm{S}\left(\frac{\bar{D}}{\mathrm{D}}\right)^{2} \tau
$$

em que, $\mathrm{S}$ - constante solar, sendo igual a $1.367 \mathrm{~W} \mathrm{~m}^{-2} ;\left(\frac{\bar{D}}{\mathrm{D}}\right)^{2}$ - expressão que permite calcular a distância relativa entre a Terra e o Sol, em razão da excentricidade da órbita terrestre, citado por 
ALVES (1981), eq.(3), e $\tau$ é a transmitância atmosférica (adimensional), apresentada por ALVES (1981), eq.(5).

$$
\left(\frac{\overline{\mathrm{D}}}{\mathrm{D}}\right)^{2}=1,000110+0,034221 \cos (X)+0,001280 \operatorname{sen}(X)+0,000719 \cos (2 X)+0,000077 \operatorname{sen}(2 X)
$$

O ângulo X, em radianos, foi calculado pela eq.(4).

$$
\mathrm{X}=\frac{2 \pi(\mathrm{DJ}-1)}{365}
$$

em que, DJ é o dia juliano, ou seja, número de dias transcorridos durante o ano, varia de 1 a 365.

$$
\tau=\exp \left[-0,089\left(\frac{P m}{1013}\right)^{0,75}-0,174\left(\frac{W m}{20}\right)^{0,60}-0,083(\mathrm{~d} \mathrm{~m})^{0,90}\right]
$$

em que, $\mathrm{P}$ - pressão atmosférica, mb; m - massa ótica da atmosfera, adimensional; W - quantidade de água precipitável na atmosfera, na direção zenital, mm, e, d - parâmetro adimensional de poeiras.

A massa óptica do ar varia com o ângulo zenital do Sol (Z), variando de 1, quando o Sol está no zênite, a 35, no nascer e pôr do Sol, sendo estimado pela eq.(6), ALVES (1981).

$$
\mathrm{m}=35\left[1224 \cos ^{2}(Z)+1\right]^{-1 / 2}
$$

A quantidade de água precipitável na atmosfera W, em altura de lâmina d'água (mm), eq.(7), ALVES (1981), foi calculada em função da pressão de vapor d'água na atmosfera (ea) medida à superfície do solo, em mb, sendo esta estimada a partir da umidade relativa e temperatura do ar.

$$
\mathrm{W}=2,5 \text { ea }
$$

O parâmetro adimensional de poeiras (d) varia de 0,2 a 3,0 conforme as condições atmosféricas reinantes, sendo, neste trabalho, adotado o valor de 0,41 , de acordo com ALVES (1981). Esta decisão deu pelo fato de ser um parâmetro de difícil mensuração e ainda, por ser o modelo muito pouco sensível à sua variação.

O ângulo de incidência da Rd com a normal à superfície $(\theta)$ foi obtido, segundo DUFFIE \& BECKMAN (1974), pela eq.(8).

$$
\begin{aligned}
\cos (\theta)= & \cos (h)[\cos (\varphi o \cos (\delta o \cos (i)+\operatorname{sen}(i) \cos (\psi \partial) \cos (\delta o \operatorname{sen}(\varphi \mathrm{e}]+\operatorname{sen}(h) \operatorname{sen}(\mathrm{i}) \operatorname{sen}(\psi \theta) \cos (\delta \mathrm{o} \\
& +\operatorname{sen}(\varphi \operatorname{esen}(\delta \operatorname{ecos}(\mathrm{i})-\operatorname{sen}(\mathrm{i}) \cos (\psi \partial) \operatorname{sen}(\delta \operatorname{ecos}(\varphi \mathrm{o}
\end{aligned}
$$

em que, h - ângulo horário, rad; $\varnothing$ - latitude do local, rad; $\delta$ - declinação solar, rad; i - ângulo de inclinação da superfície, rad, e $\Psi$ ' - azimute da superfície inclinada, tomado em relação ao Sul, variando de $0^{\circ} \mathrm{a} \pm 180^{\circ}$, positivo a oeste e negativo a leste (ALVES, 1981). dia.

O ângulo horário h, em radianos, foi determinado pela equação 9, para qualquer horário (j) do

$$
\mathrm{h}=(\mathrm{j}-12) \frac{\pi}{12}
$$


Os ângulos horários do "nascer" e do "pôr do sol", dados por $h_{1}$ e $h_{2}$, foram estimados seguindo a metodologia apresentada por ALVES et al. (1983), a qual não será aqui descrita em razão de sua extensão.

Para o uso da eq.(9), torna-se necessário reajustar o horário local (j) para o "horário solar verdadeiro", equação 10, em razão dos registros das estações meteorológicas automáticas normalmente estarem ajustados de acordo com o horário civil vigente da localidade, o qual se estende por ampla faixa geográfica, o que define os fusos horários.

$$
\mathrm{j}=\left(\operatorname{Hora}-\mathrm{FH}+\left(\frac{12}{\pi}\right) \text { long }\right)+\mathrm{Xh}
$$

em que, j - horário solar verdadeiro, h; Hora - hora local (civil) dos registros da estação meteorológica, h; FH - fuso horário civil vigente no local, h; long - longitude do local, rad, e Xh termo de correção entre a hora solar e o horário civil, devido às flutuações da duração do dia solar e a órbita terrestre. eq.(11).

Segundo a ABNT (2003), Xh é estimada em função do dia juliano (DJ), de acordo com a

$$
\mathrm{Xh}=0,170 \operatorname{sen}(1,93 \mathrm{DJ}-154,4)-0,129 \operatorname{sen}(1,01 \mathrm{DJ}-8,08)
$$

A declinação do Sol, em radianos, foi calculada pela eq.(12).

$$
\delta=0,4093 \operatorname{sen}\left(\frac{2 \pi}{365} \mathrm{DJ}-1,405\right)
$$

Estimando-se $\mathrm{I}_{0 \mathrm{i}}$, eq.(1) e demais equações complementares vistas acima, sendo esta um dado instantâneo, em $\mathrm{W} \mathrm{m}^{-2}$, torna-se necessário convertê-la para $\mathrm{kJ} \mathrm{m}^{-2}$, passando a ser, então, um valor que representa o acumulado de $\mathrm{Rd}$ interceptada num dado intervalo de tempo. Como os dados meteorológicos coletados numa estação meteorológica automática são tomados sistematicamente obedecendo a um intervalo de tempo definido, a eq.(13) é aplicada para se obter o valor de Rd em $\mathrm{kJ} \mathrm{m}^{-2}$ acumulado entre duas leituras sucessivas de dados, assumindo que a variação da radiação entre estas leituras seja linear.

$$
\mathrm{R}_{0 \mathrm{i}_{\mathrm{k}}}=\left(\mathrm{I}_{0 \mathrm{i}_{\mathrm{k}-1}}+\mathrm{I}_{0 \mathrm{i}_{\mathrm{k}}}\right) \mathrm{td} 1,8
$$

em que, $\mathrm{R}_{0 \mathrm{i}_{\mathrm{k}}}$ - Rd interceptada e acumulada durante o intervalo de tempo entre a leitura anterior ( $\mathrm{k}$ 1) e a leitura em foco $(\mathrm{k}), \mathrm{kJ} \mathrm{m}^{-2} ; \mathrm{I}_{0 \mathrm{i}_{\mathrm{k}}}$ - Rd estimada no instante em foco $\mathrm{k}, \mathrm{W} \mathrm{m} \mathrm{m}^{-2} ; \mathrm{I}_{0 \mathrm{i}_{\mathrm{k}}-1}-\mathrm{Rd}$ estimada no instante anterior ao instante em foco, $\mathrm{W} \mathrm{m}^{-2}$, e td - intervalo da duração entre os valores registrados pela estação automática, $h$. A integração entre $\mathrm{I}_{0 \mathrm{i}_{\mathrm{k}}-1}$ e $\mathrm{I}_{0 \mathrm{i}_{\mathrm{k}}}$ para td tem boa aproximação com a área de um trapézio, daí o fator 1,8 surge para a conversão da energia radiante instantânea, $\mathrm{I}_{0} \mathrm{em} \mathrm{W} \mathrm{m} \mathrm{m}^{-2}$ para o intervalo td em horas para se obter o total em $\mathrm{kj} \mathrm{m}^{-2}$.

De posse do valor de Rd interceptada e acumulada entre dois registros consecutivos de dados, pode-se fazer a acumulação da Rd durante todo o dia, eq.(14).

$$
\operatorname{Rac}_{\text {dia }}=\sum_{\mathrm{k}_{\mathrm{i}}}^{\mathrm{k}_{\mathrm{f}}} \mathrm{R}_{0_{\mathrm{i}_{\mathrm{k}}}}
$$

em que, $\mathrm{k}_{\mathrm{i}}$ - referência à primeira leitura do dia feita pela estação, e $\mathrm{k}_{\mathrm{f}}$ - referência à última leitura do dia feita pela estação. 
Contudo ainda, para se chegar ao total diário da $\mathrm{Rd}$, torna-se necessário conhecer os intervalos de exposição de cada face. Para tanto, aplicou-se a metodologia adotada por ALVES et al. (1983). Nesta metodologia, assumiu-se a superfície como sendo a própria face da linha de plantio disposta em inclinação de $90^{\circ}$ para quaisquer orientações das linhas de plantio. Foi necessário identificar o momento em que os raios solares diretos mudassem de face, ou quando os mesmos se tornam paralelos à superfície considerada. Os instantes de mudança de face, normalmente, não coincidem com os horários de leitura de dados coletados da estação meteorológica. Portanto, para melhorar a precisão das integralizações de Rd em cada face, assumiuse uma tendência linear entre os dois dados sucessivos, cujo horário de mudança de face esteja contido dentro do intervalo de ocorrência de tais dados. Conhecidos os horários sucessivos, devemse calcular os parâmetros ilustrados na Figura 1.

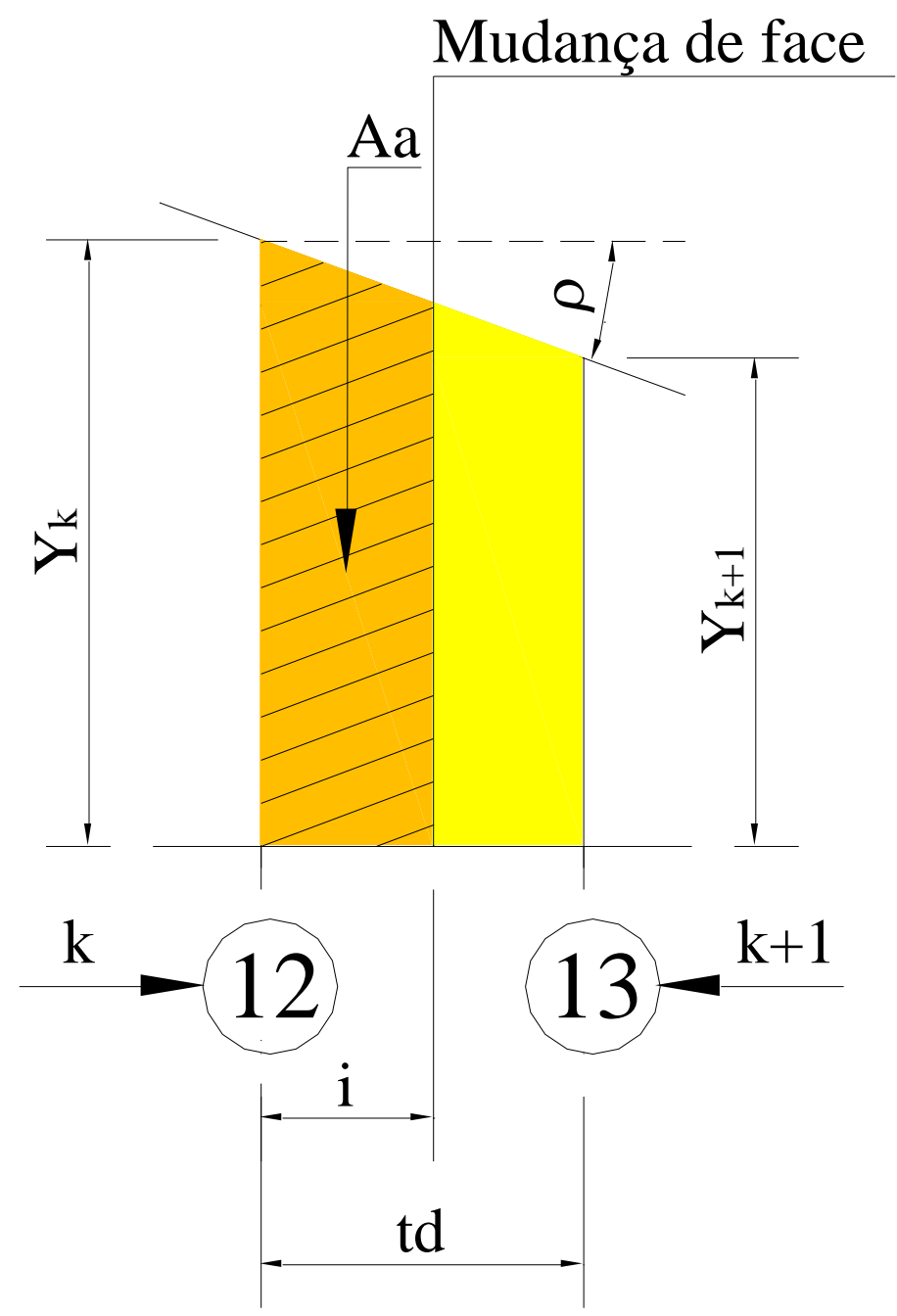

FIGURA 1. Parâmetros de ajuste para a integração da radiação solar direta na mudança de face da linha de plantio do cafeeiro (exemplo entre 12 e $13 \mathrm{~h}$ ). Adjustment parameters to the integration of the direct solar radiation in changing face of the coffee planting row (e.g. between 12 and $13 \mathrm{~h}$ ).

Na Figura 1, Y é a Rd correspondente à posição "k", determinada pela eq.(15), e $\rho$ é o ângulo formado pela reta que liga os ápices de dois valores de Y sucessivos, sendo, portando, a tangente do ângulo $\rho$, o coeficiente angular da referida reta, representado por "a", eq.(16).

$$
\mathrm{Y}_{\mathrm{k}+1}=\frac{\mathrm{R}_{0 \mathrm{i}_{\mathrm{k}+1}} 2}{\mathrm{td}}-\mathrm{Y}_{\mathrm{k}}
$$




$$
\mathrm{a}=\frac{\mathrm{Y}_{\mathrm{k}+1}-\mathrm{Y}_{\mathrm{k}}}{\mathrm{td}}
$$

A região hachurada Aa da Figura 1, eq.(17), corresponde ao que falta de $\mathrm{Rd}$ a ser integralizada como sendo interceptada por uma face da cultura após a última leitura observada e antes do momento de mudança para a outra face.

$$
\mathrm{Aa}=\int_{0}^{\mathrm{i}} \mathrm{a} \mathrm{i}+\mathrm{Y}_{\mathrm{k}} \mathrm{di}
$$

A expressão a.i $+\mathrm{Y}_{\mathrm{k}}$ é a equação a reta no intervalo entre 0 e i. $\mathrm{O}$ valor de i é dado pela eq.(18).

$$
\mathrm{i}=\mathrm{j}_{\mathrm{mud}}-\mathrm{j}_{\mathrm{k}}
$$

em que, $\mathrm{j}_{\text {mud }}$ é o horário do instante de mudança de face da linha de plantio (h) e $\mathrm{j}_{\mathrm{k}}$ é o horário referente à posição k, conforme ilustrado na Figura 1.

Os acúmulos de Rd, em um dia, para as duas faces da linha de plantio foram então calculados pelas eqs.(19) e (20).

$$
\mathrm{R}_{0_{\mathrm{f} 1 \mathrm{dia}}}=\sum_{\mathrm{ki}}^{\mathrm{kf}} \mathrm{R}_{0 \mathrm{ik}}+\mathrm{Aa}
$$

$$
\mathrm{R}_{0_{\mathrm{f} 2 \mathrm{dia}}}=\mathrm{Rac}_{\mathrm{dia}}-\mathrm{R}_{0_{\mathrm{f} 1 \mathrm{dia}}}
$$

em que, $R_{0 f 1 \text { dia }}$ e $R_{0 f 2 d i a}$ são as $R d$, interceptadas e acumuladas em ambas as faces da linha de plantio, 1 e $2, \mathrm{~kJ} \mathrm{~m}^{-2} ; \mathrm{k}_{\mathrm{i}}$ e $\mathrm{k}_{\mathrm{f}}$ são as posições que fazem referência aos instantes que delimitam o tempo de exposição de uma face da linha de plantio à $\mathrm{Rd}$.

Por fim, o acumulado de Rd interceptada por uma face da linha de plantio, durante o período diferente de um dia, é dado fazendo o somatório de $\mathrm{Rd}$ interceptada e acumulada por essa face em cada dia contido no período, eqs.(21) e (22).

$$
\mathrm{R}_{0_{\mathrm{f} 1 \text { total }}}=\sum_{\mathrm{di}}^{\mathrm{df}} \mathrm{R}_{0_{\mathrm{f} 1 \mathrm{dia}}}
$$

$$
\mathrm{R}_{0_{\mathrm{f} 2 \text { total }}}=\sum_{\mathrm{di}}^{\mathrm{df}} \mathrm{R}_{0_{\mathrm{f} 2 \mathrm{dia}}}
$$

em que, $R_{0 f 1 \text { total }}$ e $R_{0 f 2 t o t a l}$ são os totais de $R d$ interceptada e acumulada por ambas as faces da linha de plantio, em um período maior que um dia, $\mathrm{kJ} \mathrm{m}^{-2} ; \mathrm{d}_{\mathrm{i}}$ e $\mathrm{d}_{\mathrm{f}}$ são as posições que fazem referência aos dias a serem considerados para a acumulação da $\mathrm{Rd}$ interceptada.

A referência para a orientação da linha de plantio foi o norte geográfico, variando de $0^{\circ}$ a $90^{\circ}$ no sentido horário (norte a leste), e de $0^{\circ}$ a $-90^{\circ}$ no sentido anti-horário (norte a oeste). Portanto, para o cálculo de $\psi^{\prime}$, utilizado na eq.(8) para $\cos (\theta)$, tornou-se necessária a conversão da referência do presente trabalho para aquela necessária para aplicação na eq.(8), seguindo as eq.(23) e (24).

$$
\psi^{\prime}=\alpha_{\mathrm{p}}+180 \quad \text { para }-90^{\circ} \leq \alpha_{\mathrm{p}} \leq 0^{\circ}
$$

$$
\psi^{\prime}=\alpha_{\mathrm{p}}-180 \text { para } 0^{\circ} \leq \alpha_{\mathrm{p}} \leq 90^{\circ}
$$

Sendo $\alpha_{\mathrm{p}}$ a orientação da linha de plantio dado em graus.

Para a aplicação e teste do modelo proposto, elaborou-se um programa em ambiente "Borland Delphi 7" para plataforma "Windows", em linguagem "Object Pascal", cujo algoritmo simula para as possíveis orientações, conforme convenção adotada, a Rd interceptada e acumulada pelas duas faces da linha de plantio durante todo o período de avaliação. Assim, o modelo estaria indicando a orientação que promova o equilíbrio, em média, na quantidade de Rd interceptada e acumulada em ambas as faces da linha de plantio. O programa foi preparado para receber um arquivo com uma série histórica de dados de temperatura do ar, umidade relativa e pressão atmosférica, que podem ser coletados por uma estação meteorológica automática que represente o local, a fim de estimar a 
Rd interceptada pela superfície, conforme a localização, inclinação e orientação da superfície em questão.

\section{Área experimental}

Para testar o modelo, foram coletados dados de produção em uma área experimental plana de café irrigado por pivô central, na Fazenda São Thomé, em Pirapora - MG (Latitude $17^{\circ} 31^{\prime}$ 12” S, longitude $44^{\circ} 57^{\prime} 45^{\prime} \mathrm{W}$ e altitude de $520 \mathrm{~m}$ ). A lavoura de café, variedade Catuaí Vermelho 144, foi instalada em 2001 numa área circular de 80 ha, com espaçamento de 3,60 m entre linhas e 0,5 m entre plantas. Os dados de produtividade do cafeeiro analisados, em sacas de café beneficiado $\left(60 \mathrm{~kg} \mathrm{ha}^{-1}\right)$, foram do ano de 2004/2005 e, no período de $1^{\mathrm{o}}$ de janeiro a 10 de dezembro de 2004 , coletaram-se os dados de temperatura do ar, umidade relativa e pressão atmosférica registrados por uma estação meteorológica automática instalada próximo ao campo experimental.

A lavoura cafeeira em estudo possui plantio em círculos, o que dificulta o estabelecimento de um delineamento estatístico praticável em campo, para obter a casualidade e as repetições dos tratamentos. Desta forma, optou-se meramente por coletar dados de produtividade em algumas parcelas experimentais com orientação definida, sendo estas consideradas como tratamentos (Figura 2). Os tratamentos corresponderam às orientações das linhas de plantio em relação ao Norte

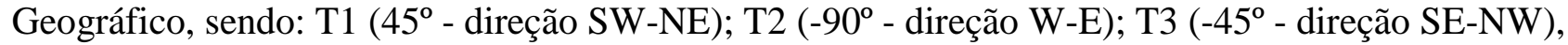
e T4 $\left(0^{\circ}\right.$ - direção S-N). Para cada tratamento, foram consideradas três linhas de plantio, em que cada uma continha três plantas úteis, e coletaram-se os frutos de café do lado externo (lado de fora em relação ao centro do pivô), lado interno (lado de dentro em relação ao centro do pivô), bem como a produção total.

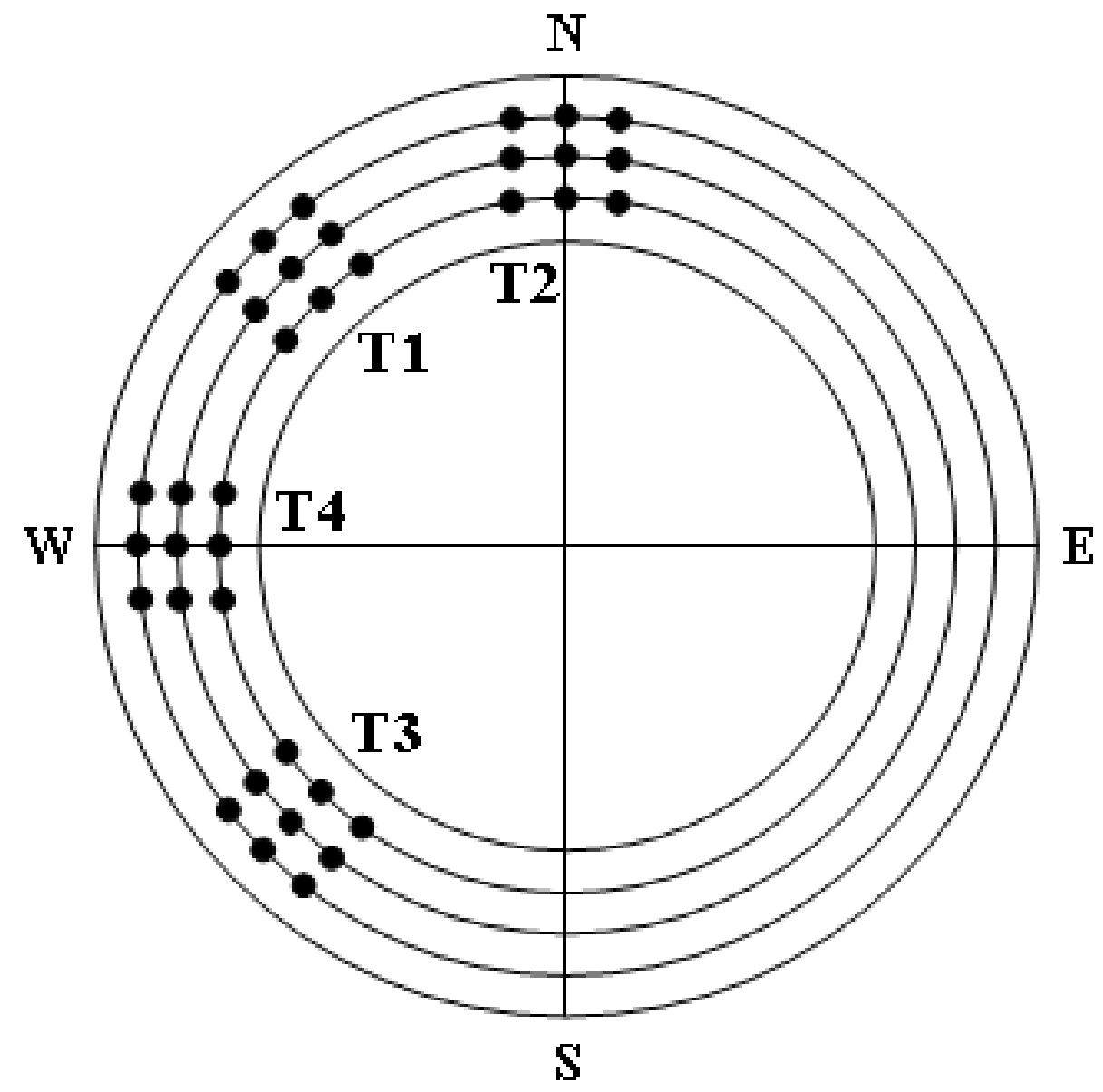

FIGURA 2. Orientação das linhas de plantio do cafeeiro em relação ao Norte Geográfico referente aos respectivos tratamentos. Orientation of the planting rows of coffee in relation to Geographic North referring to the respective treatments. 


\section{RESULTADOS E DISCUSSÃO}

Na Figura 3, está apresentada a tela de cálculo da recomendação da orientação das linhas de plantio realizada pelo programa, o qual permite, para cada área, a seleção de dados por intervalo de datas, por meses e por estações climáticas do ano. Permite, também, a indicação da precisão de cálculo, o qual se refere ao intervalo entre um ângulo e outro a ser avaliado como o ângulo adequado para a orientação das linhas de plantio. Apresenta como resultado de cálculos uma tabela com as orientações das linhas de plantio calculadas e seus respectivos valores de Rd interceptada e acumulada durante o período pela face direita e esquerda da linha de plantio e também o gráfico que esboça essas variações.

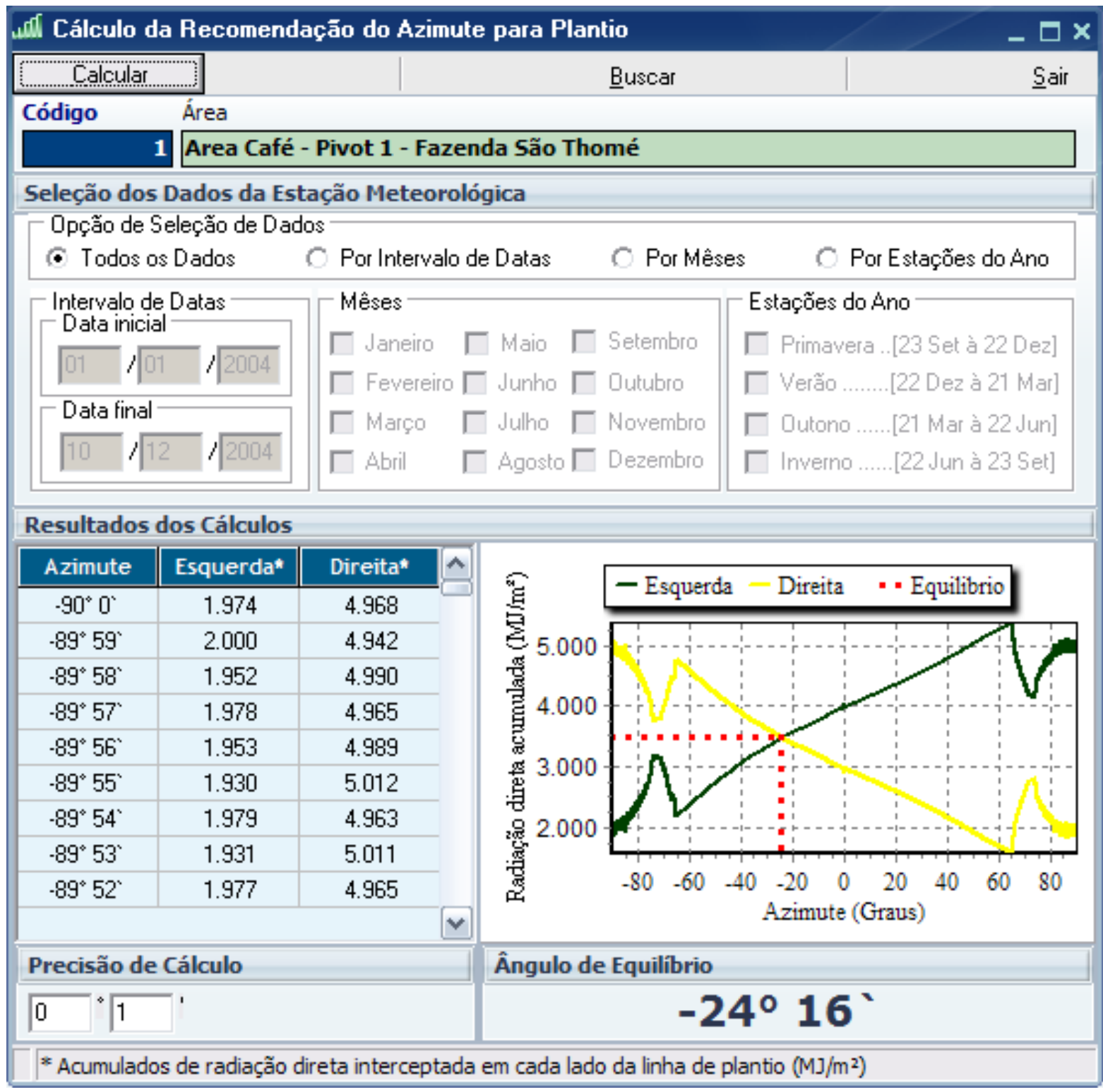

FIGURA 3. Janela do programa com apresentação do cálculo do ângulo de recomendação das linhas de plantio do cafeeiro (Ângulo de Equilíbrio). Window of the program with the presentation of the angle calculation of the recommendation of planting rows of coffee (Equilibrium Angle).

Ainda conforme é apresentada na Figura 3 e complementado pela Figura 4, para a área irrigada por pivô central da fazenda São Thomé, em Pirapora-MG, o programa gerou a orientação adequada das linhas de plantio do cafeeiro. A Figura 4 demonstra as diferentes quantidades de $\mathrm{Rd}$ interceptadas e acumuladas durante todo o período do ano de 2004, em que se coletaram os dados 
meteorológicos, para as duas faces de cada linha de plantio, sob as diferentes orientações simuladas representadas pelas duas curvas. O ponto de encontro dessas curvas indica o ângulo da orientação da linha de plantio, cujo total de Rd interceptada e acumulada seja praticamente a mesma em ambas as faces da linha de plantio, durante o ciclo de produção do cafeeiro. Portanto, nesse caso, há uma uniformidade na distribuição da $\mathrm{Rd}$ em ambas as faces da linha de plantio. E, para o presente estudo, o ângulo encontrado foi de $-24^{\circ} 16^{\prime}$, apontado pela simulação, como sendo a melhor orientação para as linhas de plantio do cafeeiro de maneira a equilibrar a quantidade de $\mathrm{Rd}$ acumulada durante o ano, em ambas as faces, de tal forma a proporcionar um equilíbrio no crescimento, desenvolvimento e produção. Este resultado difere daquele obtido por CUNHA \& VOLPE (2010), contudo são estudos um tanto diferenciados, e as coordenadas geográficas do local e relevo são altamente influentes na interceptação da radiação solar, o que favorece a diferenciação de orientações das linhas de plantio para diferentes localidades. Entretanto, devem-se realizar mais estudos desta natureza, preferencialmente em latitudes próximas, para o confronto dos resultados.

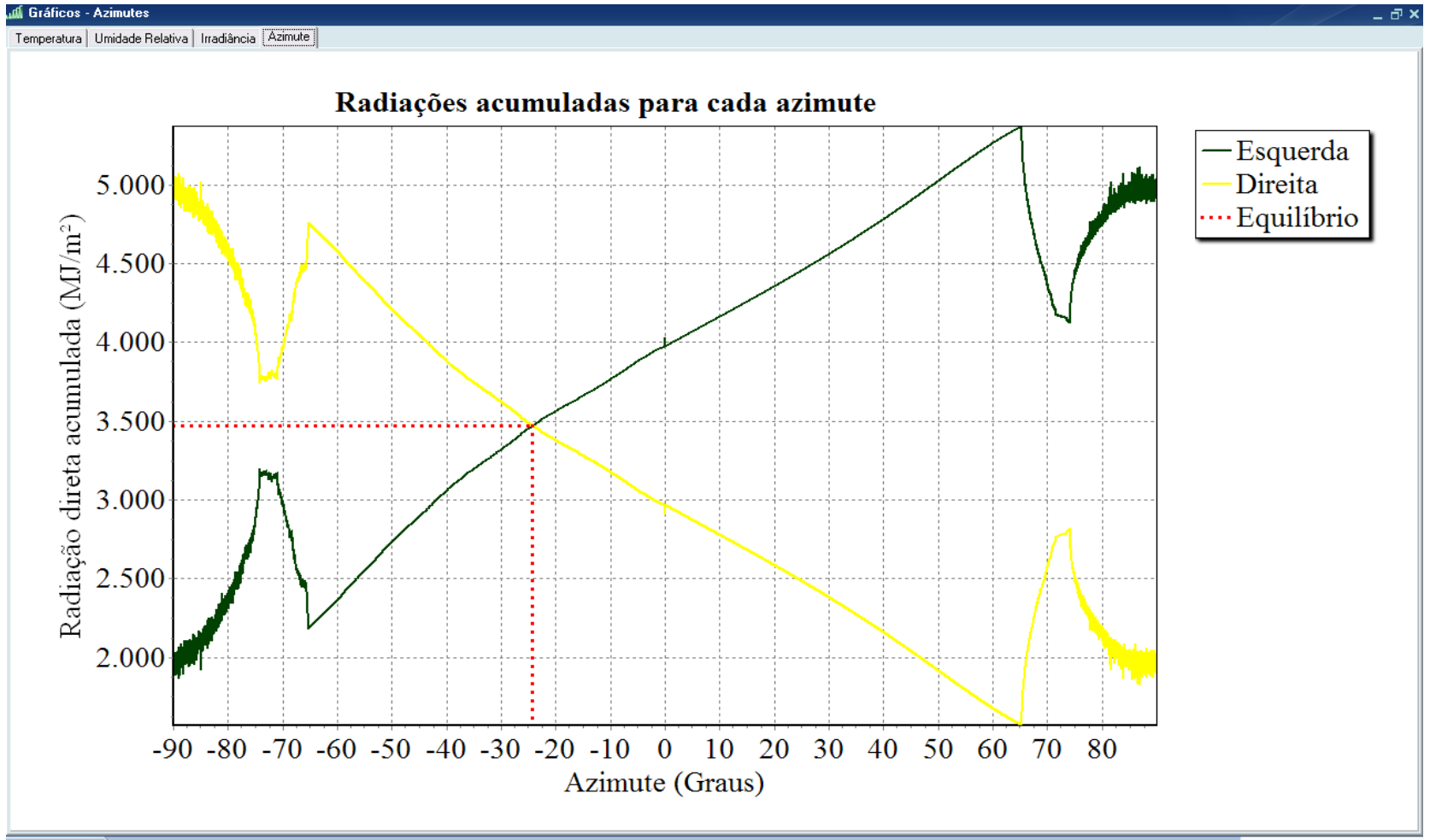

FIGURA 4. Representação gráfica da radiação solar direta interceptada e acumulada nas duas faces das linhas de plantio do cafeeiro. Graphic representation of the direct solar radiation intercepted and accumulated on both faces of the planting rows of coffee.

De acordo com a Tabela 1, na qual estão apresentados os valores de produtividade, verificou-se que a produtividade média total foi de 74,41 sacas de $60 \mathrm{~kg} \mathrm{ha}^{-1}$. Dessa, 31,45 sacas referem-se aos frutos coletados na face externa em relação ao centro do pivô, e 42,95 sacas referentes aos frutos coletados na face contrária (interna), voltada para o centro do pivô, correspondendo a 11,5 sacas a mais. Isto evidencia a influência diferenciada da interceptação da radiação solar na linha de plantio, em ambas as faces.

Ainda verificando a Tabela 1, mesmo sem análise estatística criteriosa, verifica-se que o tratamento T1 se diferenciou estatisticamente dos demais tratamentos, sendo este o que obteve as menores produtividades. Embora sem diferença significativa, o tratamento T3 foi o que obteve a maior produtividade total. 
TABELA 1. Produtividade total média em sacas $\left(60 \mathrm{~kg} \mathrm{ha}^{-1}\right)$ de café beneficiado e nas faces interna e externa em relação ao centro do pivô, em função das orientações das linhas de plantio do cafeeiro. Total yield mean in bags $\left(60 \mathrm{~kg} \mathrm{ha}^{-1}\right)$ of benefited coffee on the internal and external in relation to center pivot as a function to the orientations of planting rows of coffee.

\begin{tabular}{|c|c|c|c|}
\hline Tratamentos & Lado Externo & Lado Interno & $\begin{array}{c}\text { Produção } \\
\text { Total }\end{array}$ \\
\hline T1 (SW-NE) & 17,78 (face $S E$ ) & 25,32 (face NW) & 43,10 \\
\hline T2 (E-W) & $37,40 \quad$ (face $N)$ & $42,96$ (face $S)$ & 80,37 \\
\hline T3 (SE-NW) & $35,18$ (face $\mathrm{SW})$ & 54,53 (face NE) & 89,72 \\
\hline T4 (N-S) & $35,46 \quad($ face $W)$ & 48,98 (face $\mathrm{E}$ ) & 84,44 \\
\hline Produtividade média & 31,45 & 42,95 & 74,41 \\
\hline
\end{tabular}

Observando os dados da Tabela 1, nota-se que as plantas, seguindo a orientação das linhas de plantio SW-NE, ou seja, com as respectivas faces para o SE e NW, são as de menor produtividade, enquanto as plantas seguindo a orientação SE-NW, cujo ângulo é de $-45^{\circ}$, portanto com face NE e $\mathrm{SW}$, são as que mais produziram. Este resultado vem corroborar a orientação da linha de plantio estimada no presente trabalho, cujo valor recomendado foi de $-21^{\circ} 16^{\prime}$.

Quanto à distribuição da Rd, a direção SE-NW foi a que proporcionou maiores produtividades, embora havendo certo desequilíbrio da produtividade em relação às faces da linha de plantio. Pode-se inferir que esse resultado esteja relacionado com que a direção ideal de plantio é aquela em que as plantas sejam orientadas de tal modo que, durante o período do dia de maiores temperaturas, que corresponde à tarde, estejam expostas ao Ssol por um tempo inferior ao que foram expostas pela manhã, quando a temperatura é mais amena.

\section{CONCLUSÕES}

O modelo apresentado permite determinar, com precisão satisfatória, a orientação adequada das linhas de plantio do cafeeiro, com base na homogeneidade da interceptação da radiação solar direta em ambas as faces da linha de plantio.

Os resultados obtidos pela modelagem apresentada foram satisfatórios quando comparados com resultados de produtividade obtidos em campo.

\section{AGRADECIMENTO}

Ao Conselho Nacional de Desenvolvimento e Pesquisa - CNPq, pela concessão de bolsa ao primeiro autor, durante o curso de mestrado em Engenharia Agrícola - Engenharia de Água e Solo, pela Universidade Federal de Lavras.

\section{REFERÊNCIAS}

ABNT. ASSOCIAÇÃO BRASILEIRA DE NORMAS TÉCNICAS. Iluminação natural - parte 2: procedimentos de cálculo para estimativa da disponibilidade de luz natural. Rio de Janeiro, 2003. $17 \mathrm{p}$.

ALVES, A.R. Irradiância solar global em superfícies de diferentes inclinações e azimutes, para Viçosa-MG. 1981. 92 f. Dissertação (Mestrado) - Universidade Federal de Viçosa, Viçosa, 1981.

ALVES, A.R.; VIANELLO, R.L.; SEDIYAMA, G.C.; COELHO, D.T. Determinação analítica dos instantes do "nascer" e do "pôr do sol" para superfícies inclinadas quaisquer. Revista Ciência e Cultura, São Paulo, v.35, n.2, p.194-198, 1983. 
ANGELOCCI, L.R.; MARIN, F.R.; PILAU, F.G.; RIGHI, E.Z.; FAVARIN, J.L. Radiation balance of coffee hedgerows. Revista Brasileira de Engenharia Agrícola e Ambiental, Campina Grande, v.12, n.3, p.274-281, 2008.

BOSCH, J.L.; LÓPES, G.; BATLLES, F.J. Global and direct photosynthetically active radiation parameterizations for clear-sky conditions. Agricultural and Forest Meteorology, Amsterdam, v.149, p.146-158, 2009.

BRAUN, H.; ZONTA, J.H.; LIMA, J.S. de S.; REIS, E.F. dos. Produção de mudas de café 'conilon' propagadas vegetativamente em diferentes níveis de sombreamento. IDESIA, Chile, v.25, n.8, p.8591, 2007.

BROOKS, F.A. An introduction to physical microclimatology. Davis: University of California, 1959. $264 \mathrm{p}$.

CUNHA, A.R. da; VOLPE, C.A. Relações radiométricas no terço superior da copa de cafeeiro. Bragantia, Campinas, v.69, n.2, p.263-271, 2010.

CONAB. COMPANHIA NACIONAL DE ABASTECIMENTO. Acompanhamento da Safra brasileira café - Safra 2010 segunda estimativa, maio/2010. Brasília, 2010. 18 p.

DONATELLI, M.; CARLINI, L.; BELLOCCHI, G. A software component for estimating solar radiation. Environmental Modelling \& Software, Oxford, v.21, p.411-416, 2006.

DUFFIE, J.A.; BECKMAN, W.A. Solar energy thermal processes. New York: John Wiley e Sons, 1974. $386 \mathrm{p}$.

GANIS, A. Radiation transfer estimate in a row canopy: a simple procedure. Agricultural and Forest Meteorology, Amsterdam, v.88, p.67-76, 1997.

LIMA, E. de A.; MOLION, L.C.B.; GOMES FILHO, M.F.; FIRMINO, J.L. da N.; SILVA, A.O. da. Variabilidade interanual da profundidade óptica da atmosfera sobre Maceió-AL. Revista Brasileira de Engenharia Agrícola e Ambiental, Campina Grande, v.11, n.5, p.509-514, 2007.

OLIPHANT, A.; SUSAN, C.; GRIMMOND, B.; HANS-PETER SCHMID; WAYSON, C.A. Local-scale heterogeneity of photosynthetically active radiation (PAR), absorbed PAR and net radiation as a function of topography, sky conditions and leaf area índex. Remote Sensing of Environment, New York, v.103, p.324-337, 2006.

OLIVEIRA, E.L.; FARIA, M.A.; REIS, R.P.; SILVA, M.L.O. Manejo e viabilidade econômica da irrigação por gotejamento na cultura do cafeeiro acaiá, considerando seis safras. Engenharia Agrícola, Jaboticabal, v.30, n.5, p.887-896, 2010.

PALTRIDGE, G.W.; PLATT, C.M.R. Radiative processes in meteorology and climatology. Amsterdam: Elsevier Scientific, 1976. 318 p.

PEZZOPANE, J.R.M.; PEDRO JÚNIOR, M.J.; GALLO, P.B. Caracterização microclimática em cultivo consorciado café/banana. Revista Brasileira de Engenharia Agrícola e Ambiental, Campina Grande, v.11, n.3, p.256-264, 2007.

PEZZOPANE, J. R. M.; PEDRO JÚNIOR, M.J.; GALLO, P.B. Radiação solar e saldo de radiação em cultivo de café a pleno sol e consorciado com banana 'Prata-Anã'. Bragantia, Campinas, v.64, n.3, p.485-497, 2005.

RIGHI, C.A.; BERNARDES, M.S.; LUNZ, A.M.P.; PEREIRA, C.R.; DOURADO NETO, D.; FAVARIN, J.L. Measurement and simulation of solar radiation availability in relation to the growth of coffee plants in an agroforestry system with rubber trees. Revista Árvore, Viçosa-MG, v.31, n.2, p.195-207, 2007.

SANTINATO, R.; FERNANDES, A.L.T.; FERNANDES, D.R. Irrigação na cultura do café. Campinas, 1996. 146 p. 
TATAGIBA, S.D.; PEZZOPANE, J.E.M.; REIS, E.F. dos. Crescimento vegetativo de mudas de café arábica (Coffea arabica L.) submetidas a diferentes níveis de sombreamento. Coffee Science, Lavras, v.5, n.3, p.251-261, 2010.

TOVAR-PESCADOR, J.; POZO-VÁZQUEZ, D.; RUIZ-ARIAS, J.A.; BATLLES, J.; LÓPEZ, G.; BOSCH, J.L. On the use of the digital elevation model to estimate the solar radiation in areas of complex topography. Meteorological Applications, Reading, v.13, p.279-287, 2006.

TURCO, J.E.P.; RIZZATTI, G.S. Avaliação de modelo matemático para estimar a radiação solar incidente sobre superfícies com diferentes exposições e declividades. Engenharia Agrícola, Jaboticabal, v.26, n.1, p.257-267, 2006. 